\title{
Modernidad en la patrimonial ciudad de Quito
}

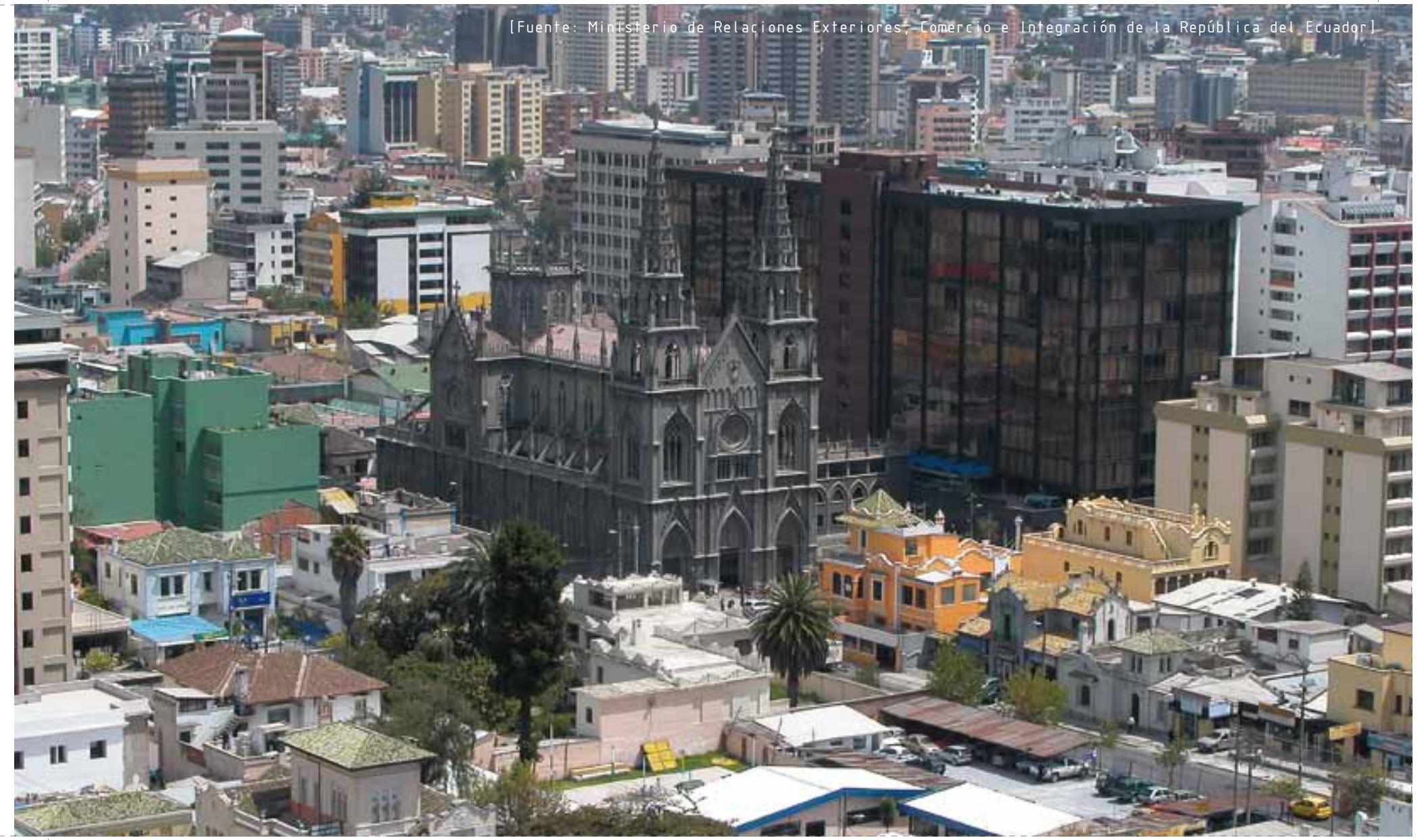

Registrar la modernidad en Quito, Patrimonio Cultural de la Humanidad, es una faena sugerente, y para un foráneo, todo un descubrimiento. La imagen mental preexistente, restringida al carácter patrimonial colonial, se renueva gracias al encuentro con una arquitectura moderna que es fruto de procesos económicos y sociales que acaecieron principalmente a partir de mediados de la década del 60
Hasta finales de los 50 Quito se reducía a barrios tradicionalmente coloniales. Es a principios de los 60, debido al impulso de la industrialización y la empresa privada por parte del Estado, que comienzan a emplazarse los primeros edificios modernistas al norte del centro histórico. En esta década y la siguiente, potenciada por el «boom petrolero", la Arquitectura Moderna tiene su mayor desarrollo abarcando edificios estatales, institucionales, infraestructura pública, viviendas, edificios para las cámaras profesionales, centros comerciales y edificios privados

\section{El presente registro fotográfico pretende} rescatar 5 edificios emblemáticos de la época, debido a su funcionalidad, sistema constructivo, estética, importancia histórica y, en uno de los casos, su lamentable abandono.

* Arquitecto de la Universidad de Chile, 2005. Diplomado en Políticas Públicas, PUC 2006. Actualmente desarrolla labores de voluntario en Guayaquil. Ecuador. 


\section{EDIFICIO ARTETA (PHILIPS)}

1955

Arqto. Lionel Ledesma

Con una planta de $160 \mathrm{~m}^{2}$ de oficinas

privadas por nivel y un primer piso destinado a comercio es de los primeros edificios con este carácter funcional en Quito: oficinas privadas y comercio.

Construido en hormigón armado destaca la expresión de las distintas fachadas por composición en distintos planos, su asimetría y su rico juego de llenos y vacios.
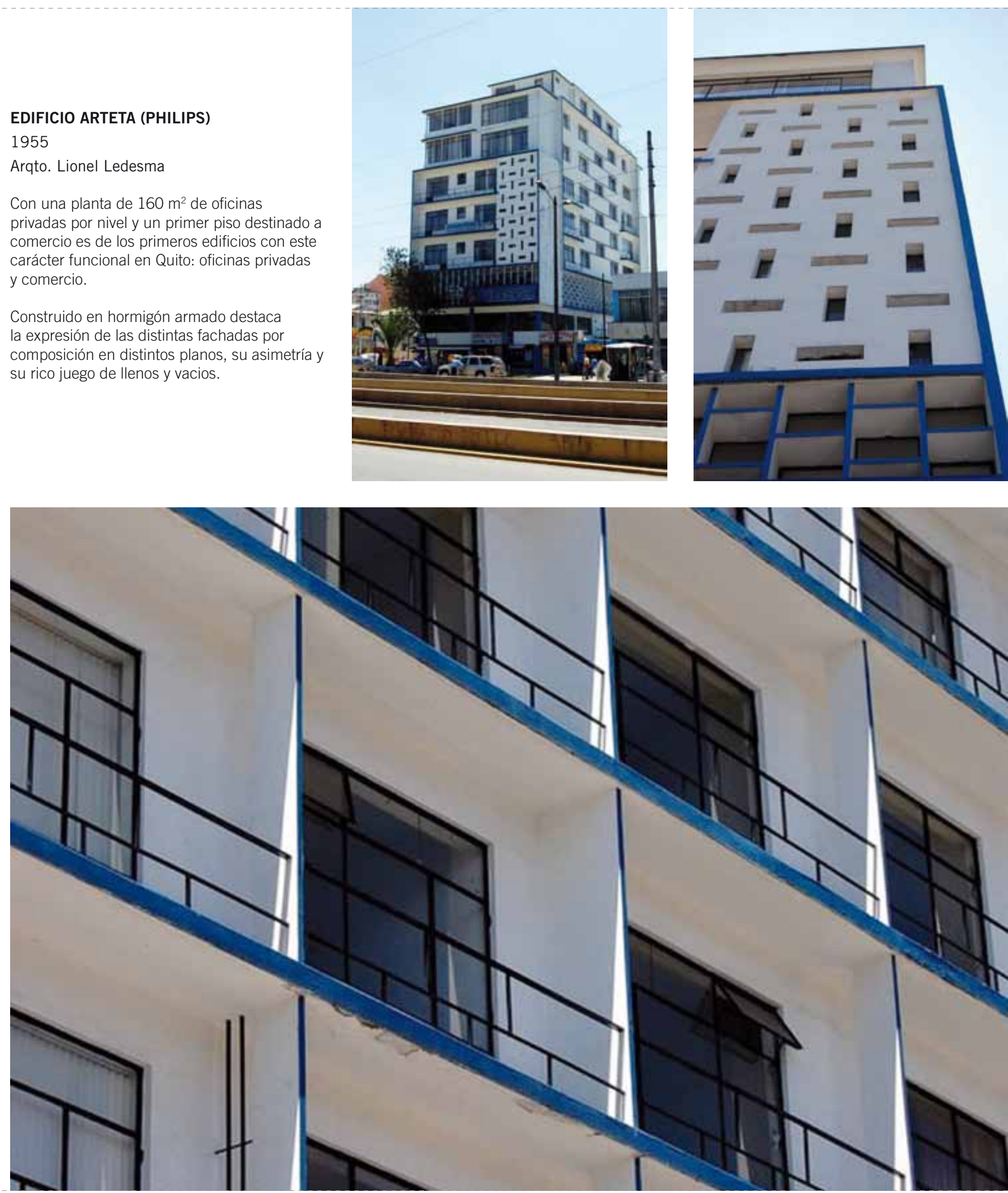
RESIDENCIA UNIVERSITARIA DE LA UNIVERSIDAD CENTRAL DEL ECUADOR 1960

Arqto. Mario Arias Salazar

Concebido como residencia universitaria, de sus dependencias, este edificio destaca por su ortodoxia al momento de plasmar los planteamientos del movimiento moderno.

Bajo el esquema del pabellón de Marsella el planta libre sobre pilotes, la disposición libre sobre el sitio, la racionalización de elementos lineales y constructivos, la cubierta habitable, cuerpos independientes para circulaciones verticales y un emplazamiento sur-norte que busca el aprovechamiento del sol. reutilizado como Hospital para la universidad y hoy en lamentable abandono la mayoría edificio se desarrolla bajo la concepción de la
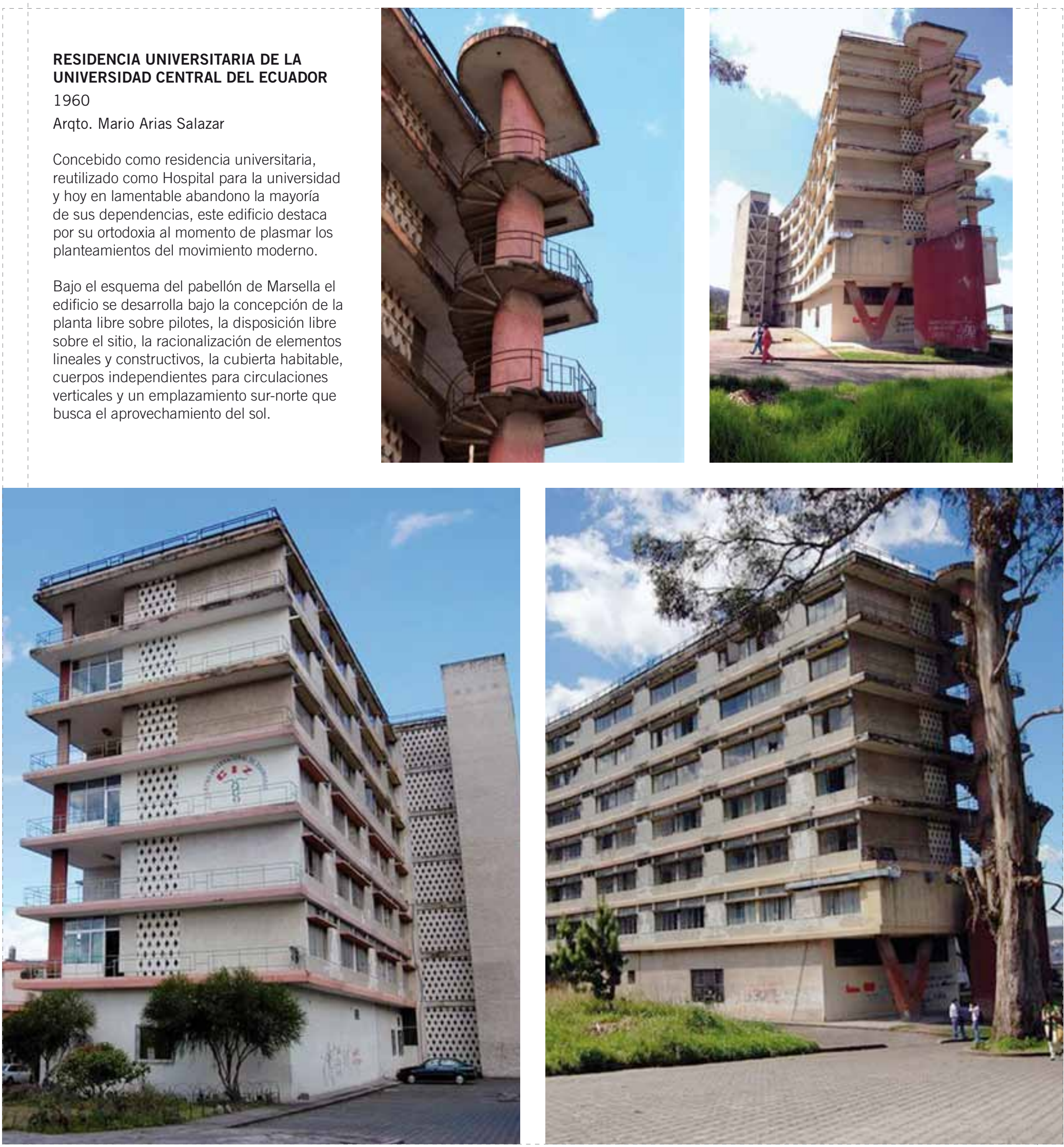
CASA DE LA CULTURA ECUATORIANA

1956-1960 Anteproyecto

y Proyecto Definitivo

1975-1980 Construcción

Arqto. René Denis Zaldumbide

Edificio de planta ovoidal, estéticamente rupturista y con gran presencia urbana se configura como un hito dentro de un tejido urbano predominantemente natural y patrimonial para su época.

Destaca la composición formal a partir de 3 elementos característicos: la fachada de acceso construida a partir de distintos elementos en hormigón armado, el resto del edificio revestido con un muro cortina acristalado y la techumbre constituida por distintos planos hiperbólicos.
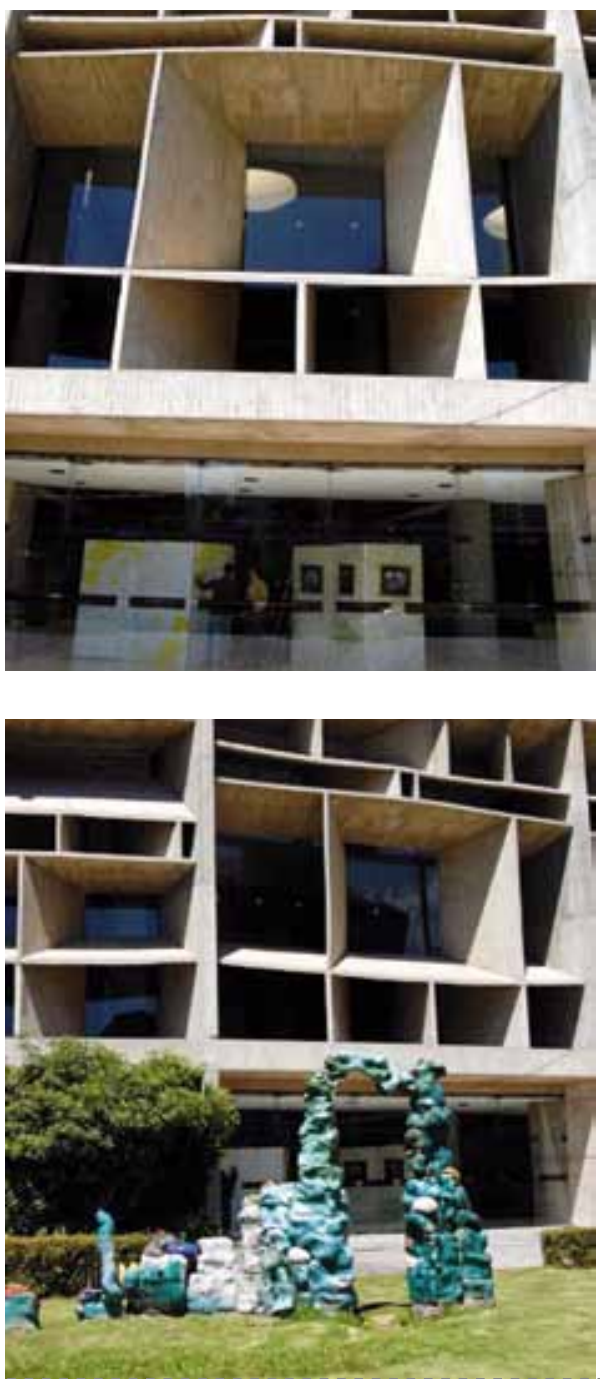
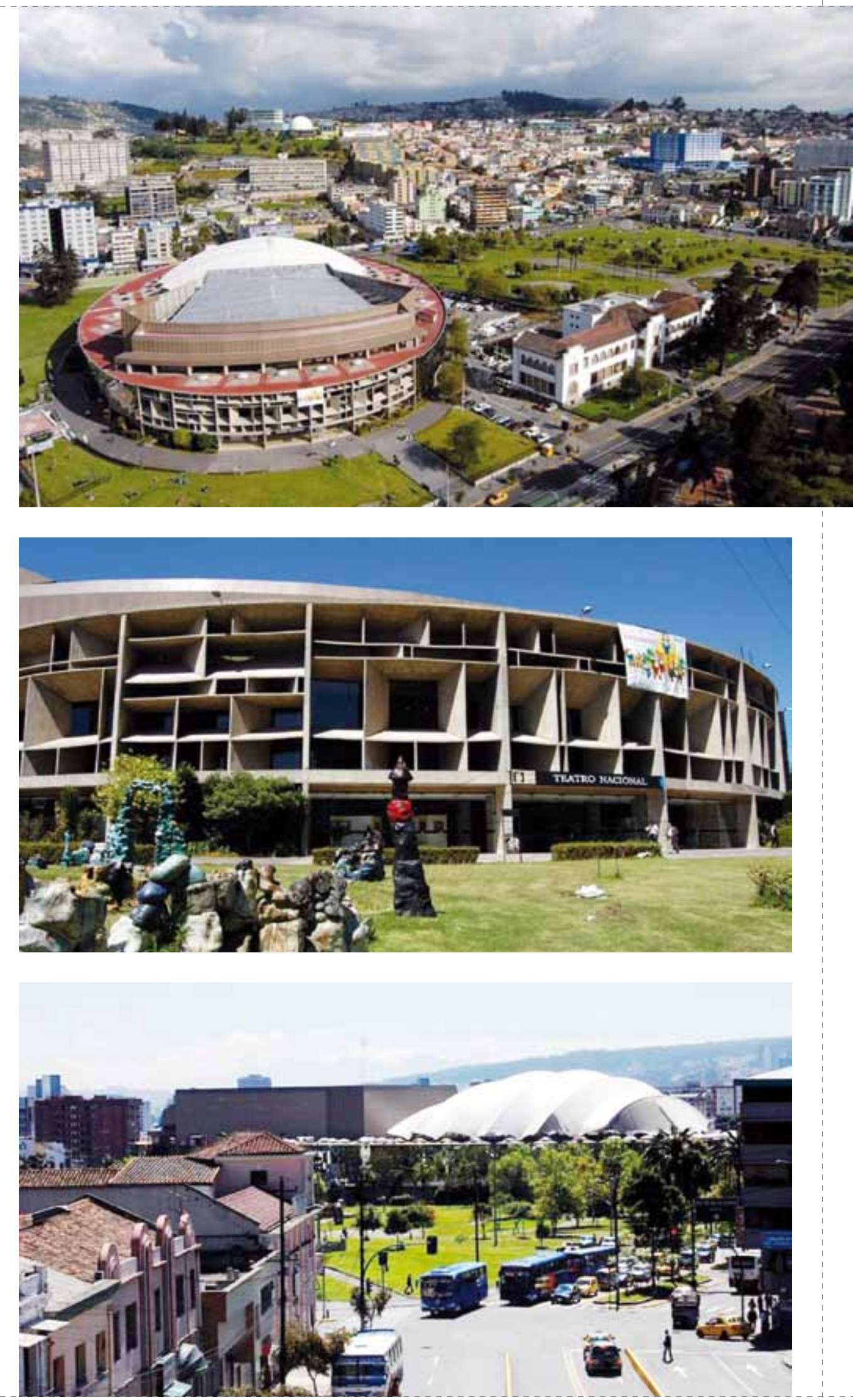


\section{CONDOMINIO MAÑOSCA}

1967

Arqto. Rubén Moreira

Este conjunto de 80 departamentos

desarrollado para el Banco de la Vivienda fue el primero de carácter social construido en la ciudad. Innovador no sólo por su uso, en este edificio se utiliza por primera vez mampostería estructural con columnas de arriostramiento de $20 \mathrm{~cm}$ por $20 \mathrm{~cm}$.

Además por primera vez se utiliza el concepto de coordinación modular, dimensionando las plantas con un modulo de $40 \mathrm{~cm}$, que era la medida de los bloques de cemento de la época, lo que se traduce en luces estructurales de $3,20 \mathrm{~m}$.
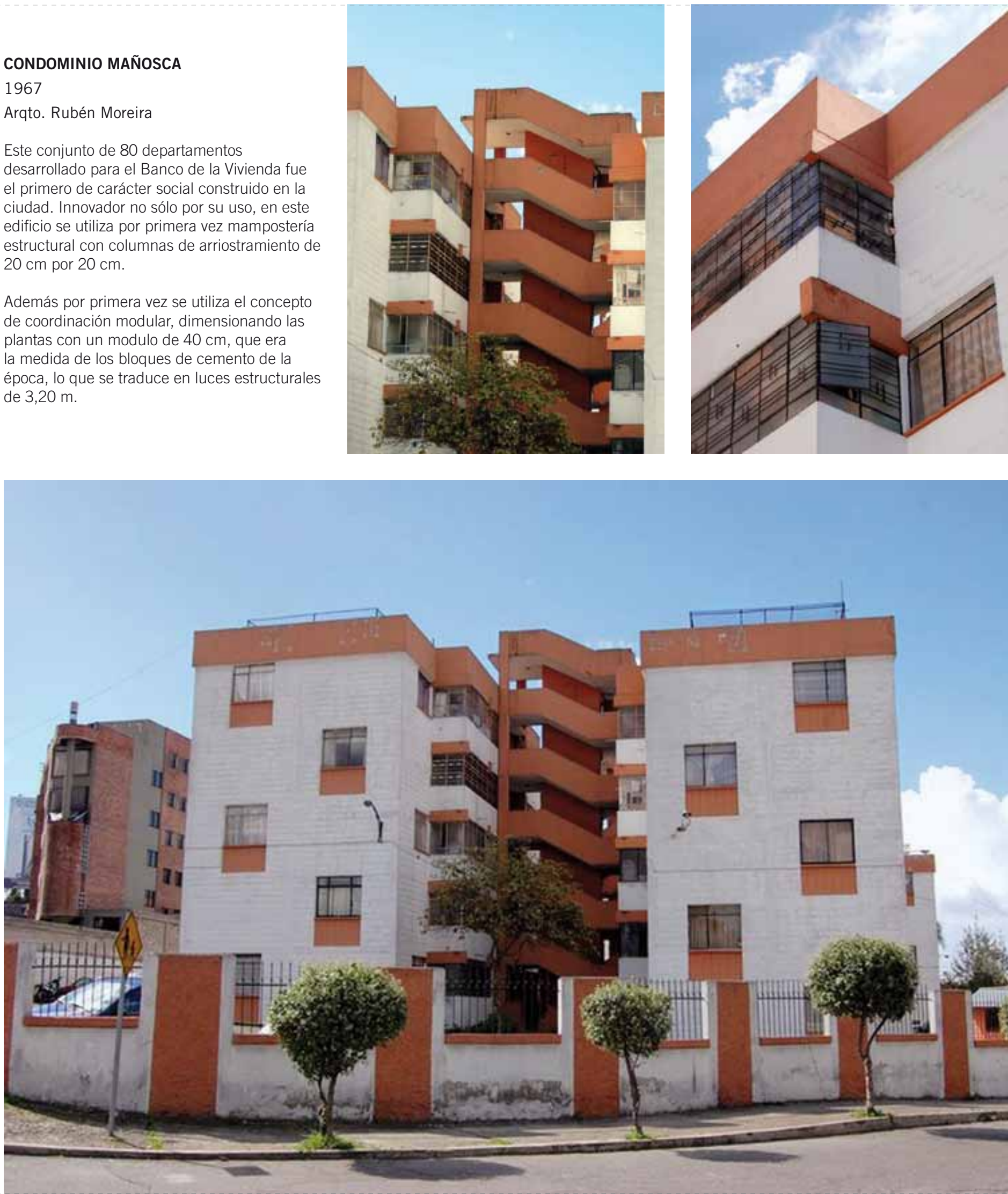


\section{EDIFICIO CFN (CORPORACIÓN} FINANCIERA NACIONAL)

\section{4}

Arqto. Ovidio Wappenstein

Arqto. Ramiro Jácome

Arqto. Colaborador César Gálvez

De nítida impronta brutalista, una placa en el primer piso y planta ligeramente ochavada, es un indiscutible emblema de la arquitectura moderna en el Quito de los años '70. Gran reflejo de la importancia social y política de las instituciones financieras de la época.

El edificio más alto de Quito hasta la actualidad, se destaca por su vocación urbana la que se potencia debido al parque que lo antecede. Esta vocación lo transforma en componente emblemático de la imagen urbana del Quito moderno.

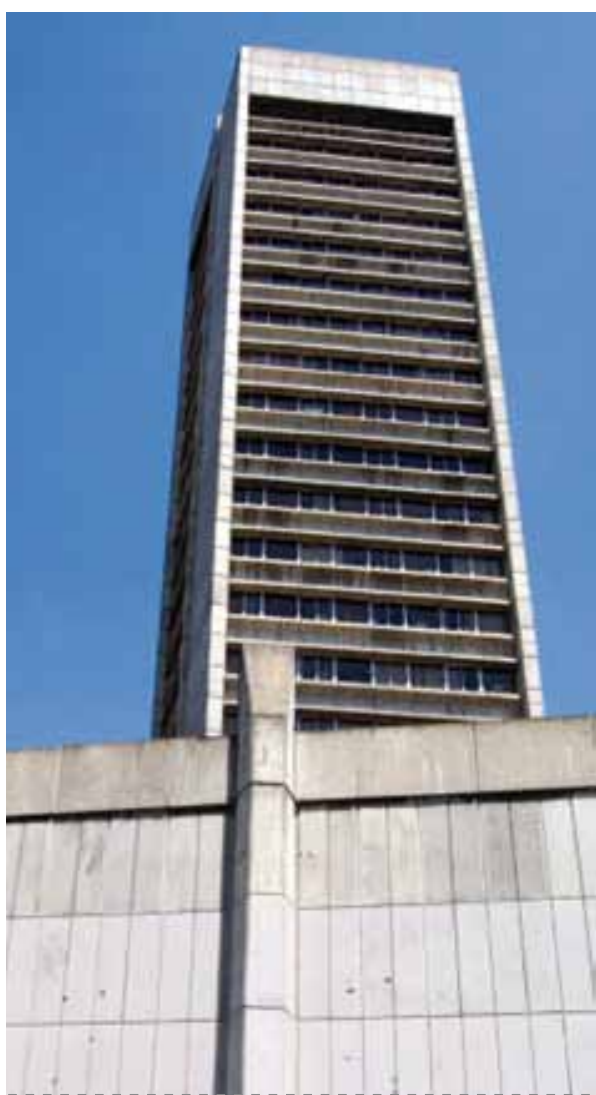

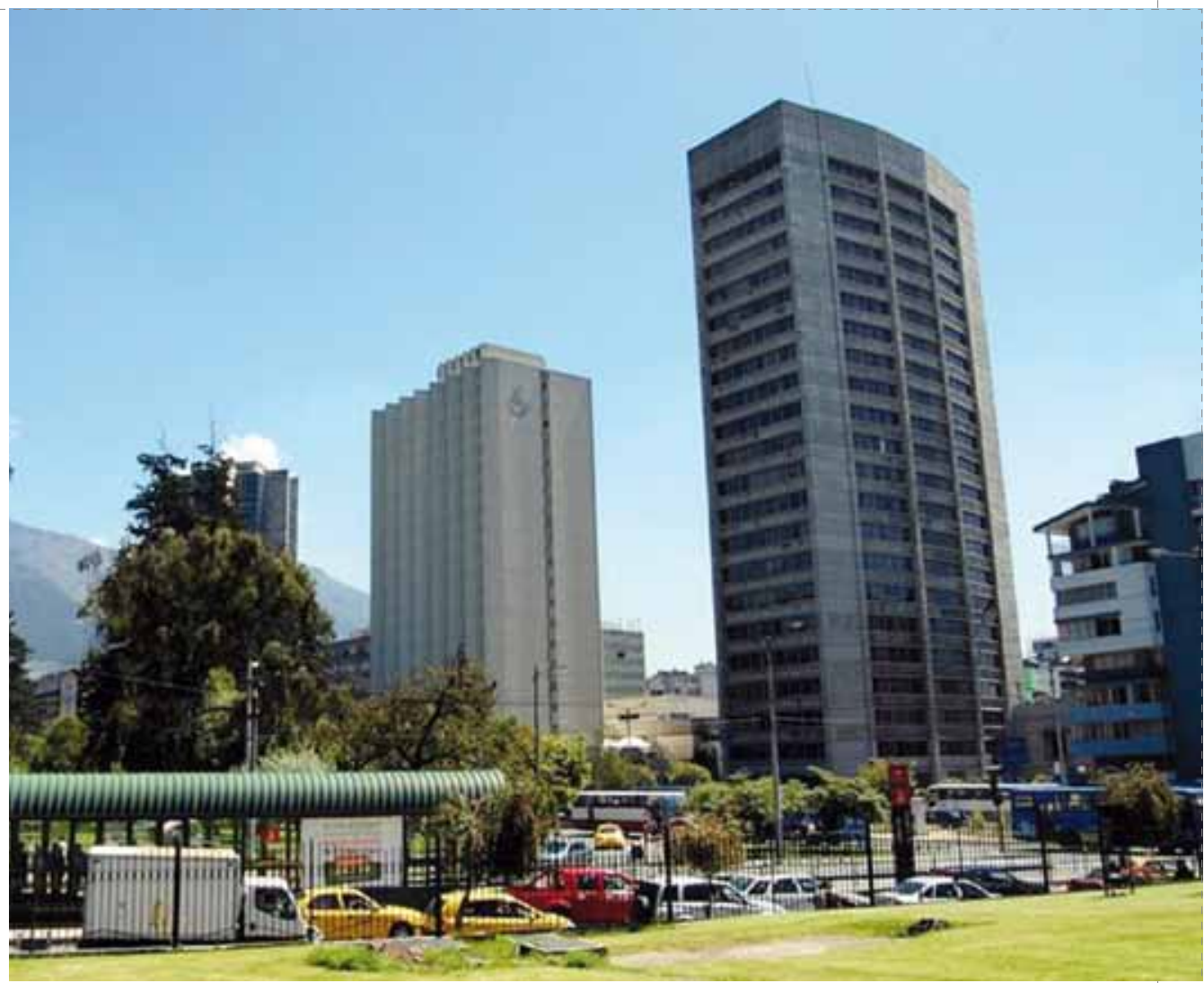

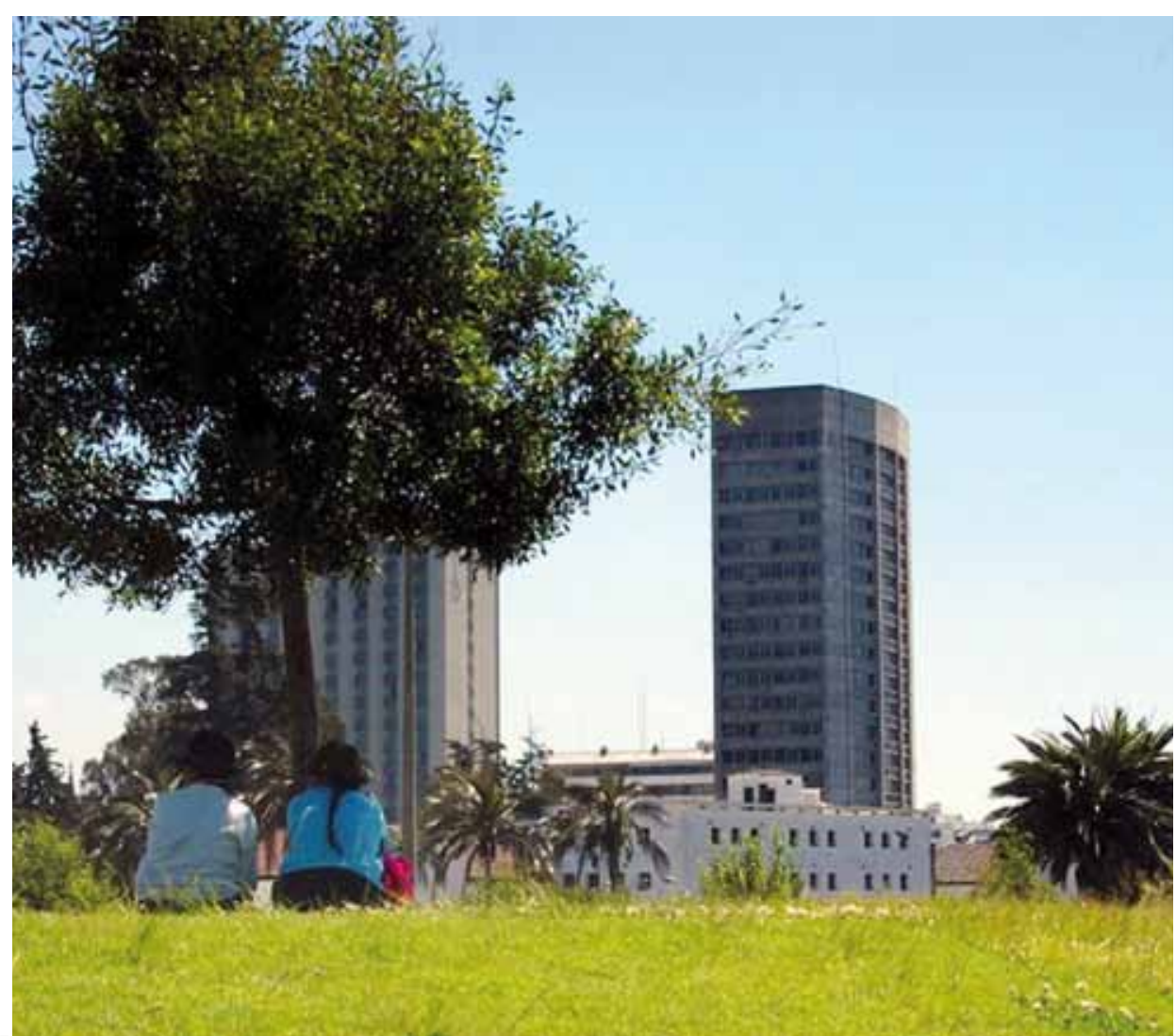

